دراسة التأثير السمي للكولسينات الخام الحرة (غير المرتبطة) المستخلصة من فئن

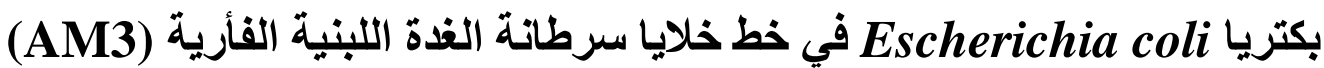
المغروسة في الفئران المختبرية الطبيعية

\title{
Study the toxic effect of non-bound colicins extracted from Escherichia coil on transplanted Murine adenocarcinoma $\left(\mathrm{AM}_{3}\right)$
}

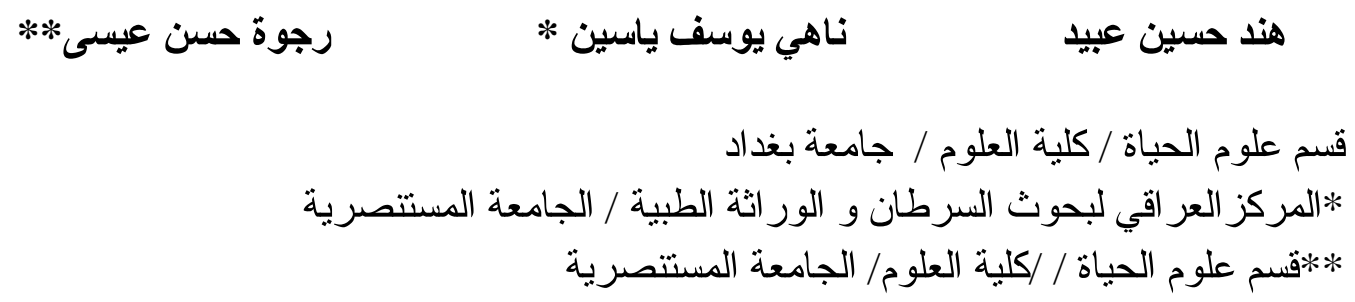

\section{Hind Hussein Obaid Nahiy Yousif Yaseen* Rajwa Hassan Essa **}

Dept. of Biology/ College of Science /Baghdad University

* Iraqi Center of Cancer and Genetic medicine/ Al-Mustansiyria University ** Dept. of Biology/College of Science/ Al-Mustansiyria University

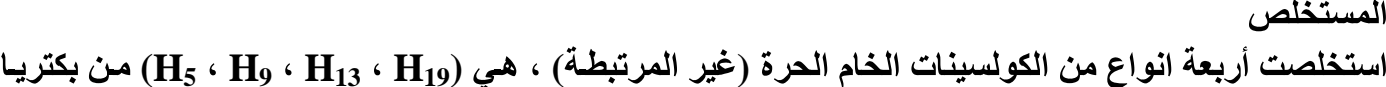
E.coli

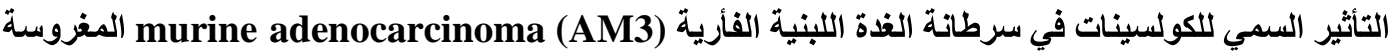

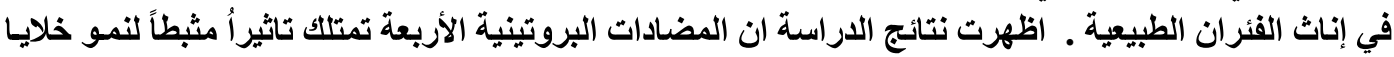

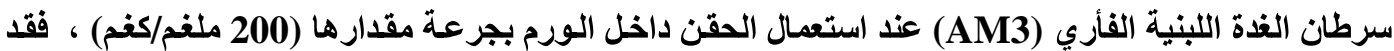

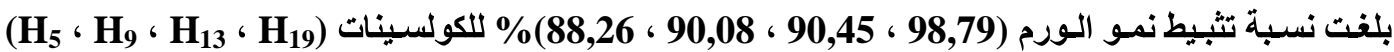

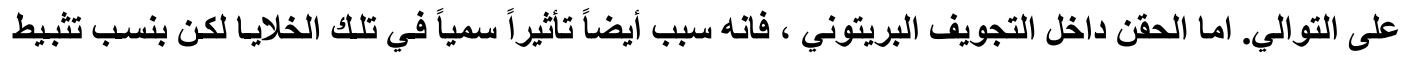

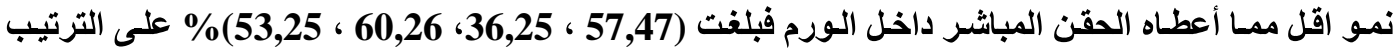

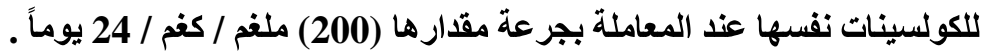

Abstract

Four types of non-bound colicins $\left(\mathrm{H}_{5}, \mathrm{H}_{9}, \mathrm{H}_{13}, \mathrm{H}_{19}\right)$ extracted from intestinal normal flora E.coli isolated from apparently healthy people's stool. Then cytotoxic effect of colicins on transplanted murine adenocarcinoma (AM3) was studied. The results showed,that the four colicins had inhibitory effects on growth of AM3 cells when injected intratumorally at $(200) \mathrm{mg} / \mathrm{kg}$. Percentages of tumor inhibition were (98.79, 90.45, 90.08 and 88.26) \% for colicins $\left(\mathrm{H}_{19}, \mathrm{H}_{13}, \mathrm{H}_{9}\right.$ and $\left.\mathrm{H}_{5}\right)$ respectively. Intraperitoneal injection caused less inhibition, $(57.47,36.25,60.26$ and 53.25$) \%$ respectively using a daily dose of $(200) \mathrm{mg} / \mathrm{kg}$ for period of 24 days.

Key Words: E.coli , colicins, cytotoxicity , murine adenocarcinoma, AM3 . 


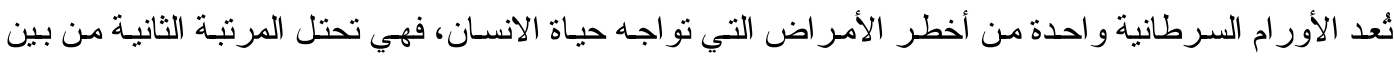

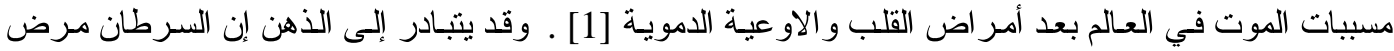

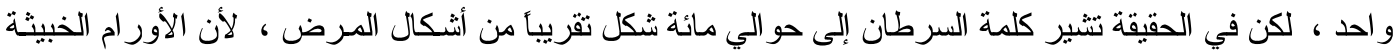

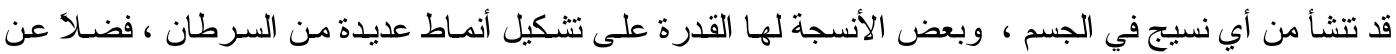

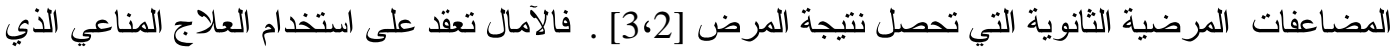

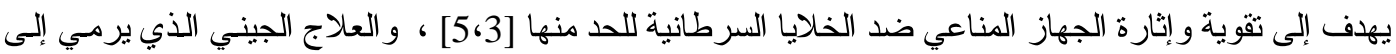

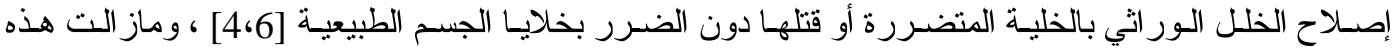

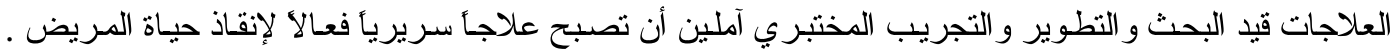

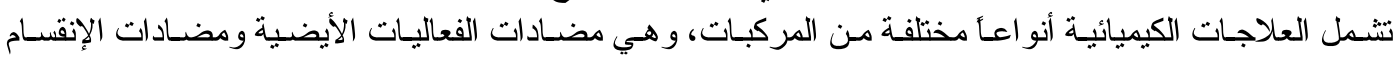

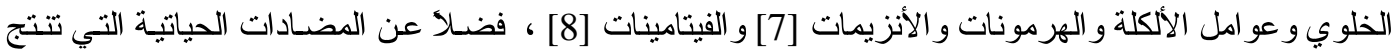

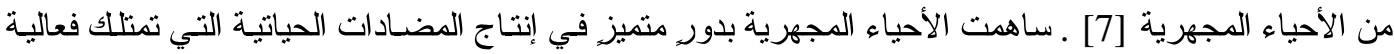

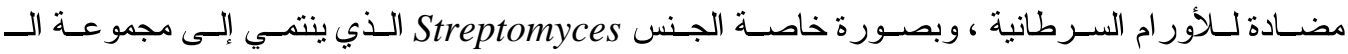
لعال Actinomycetes

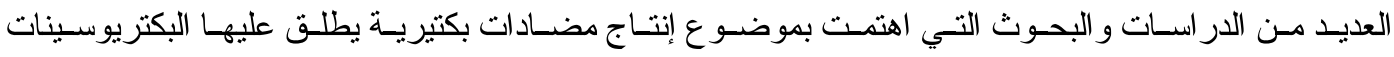

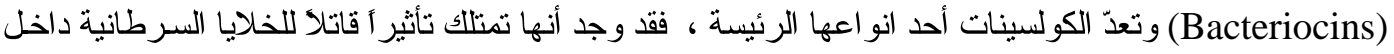

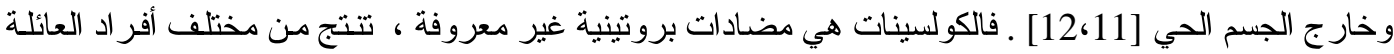
المعويـة (Enterobacteraceae) وبصورة رئيسـة من بكتيريـا Escherichia coli وتنتكن من قتنل الأنواع

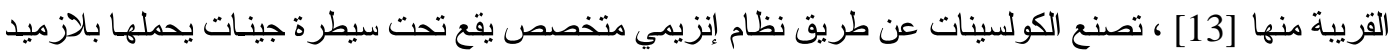

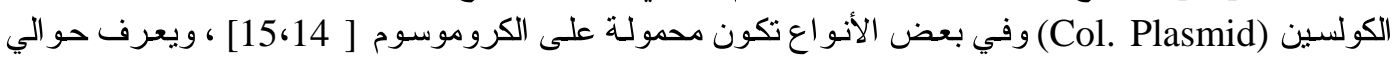

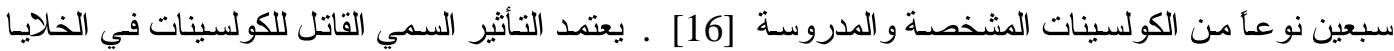

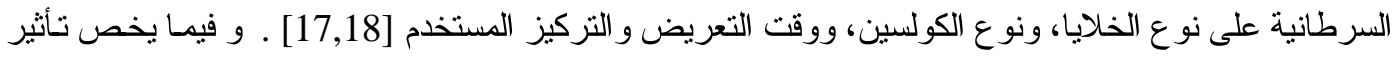

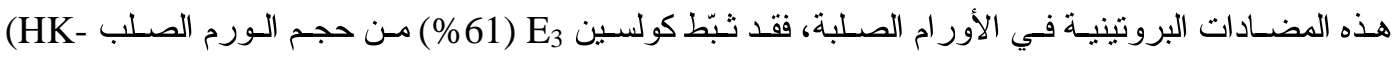

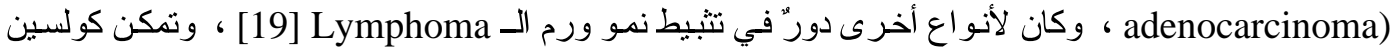
HSC 10

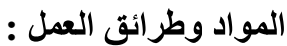

استخلاص الكولسينات الخام الحرة : (غير المرتبطة)

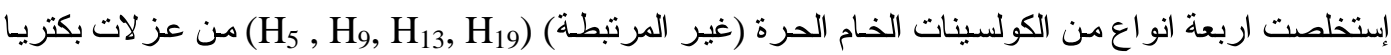

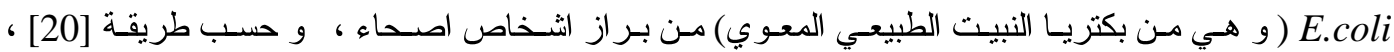

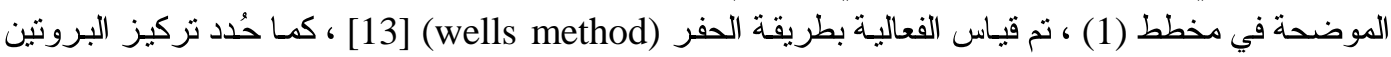
الكلي [21] . 
مخطط (1) : حث و استخلاص الكولسينات الخام الحرة

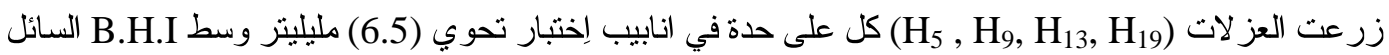

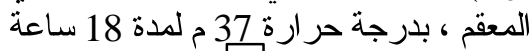

حضر وسط (B.H.I السائل + 5\% كليسرول) في دو ارق مناسبة الحجم ، بو اقع (250) مليليتر في كل دورق ، و عقمت بالموصدة و ونركت لتبرد

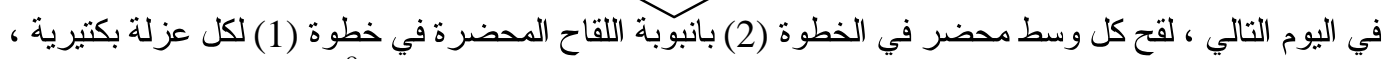

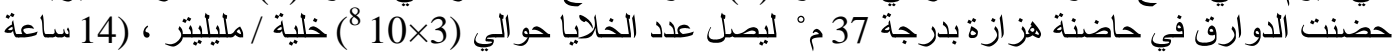

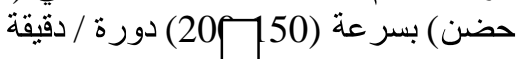

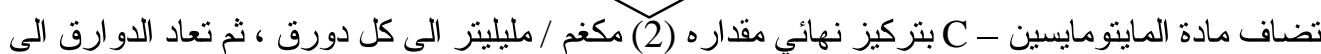
الحاضنة الهز ازة لمدة (3) ساعات (2كنات إضافية

بعد انتهاء فترة الحضن ، نبذ المزروع البكتيري لكل دورق على إنفصال بجهاز الطرد المركزي المبرد بدرجة حر ارة

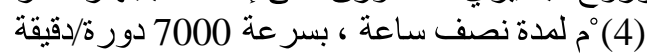

فصل الر اشح عن الر اسب ، و عدت الرو اثح المحضرة هي الكولسينات الخام الحرة ، قيست الفعالية و تركيز البروتين في كل نو عنم منها

دراسة التأثير السمي للكولسينات في خط خلايـا سرطانة الغدة اللبنية الفاريـة (AM3) المغروسـة في الفئران

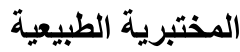

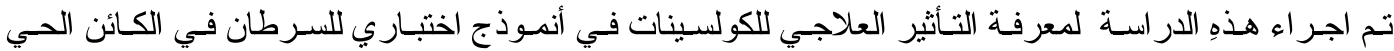
(Animal Model System) أولاً: غرس الخلايا السرطانية

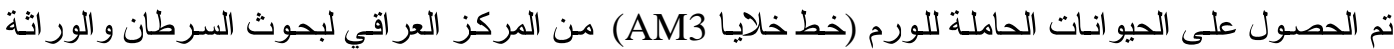

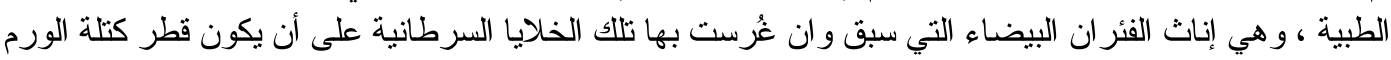

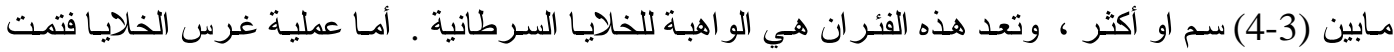
كالآتي:

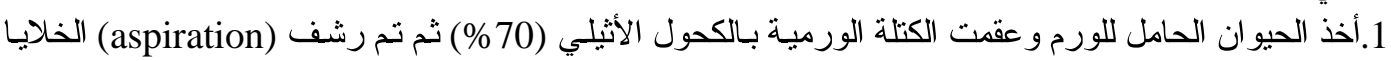
السرطانية من تللك الكتلة باستعمال محقنة معقمة عن طريق ادخال إبرة ذات قياس (18) لنحصل على حو الي (35) مل من الخلايا السرطانية.

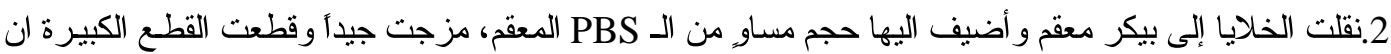

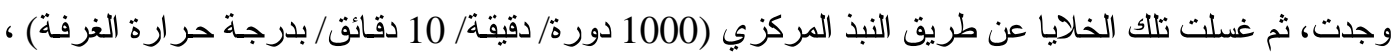

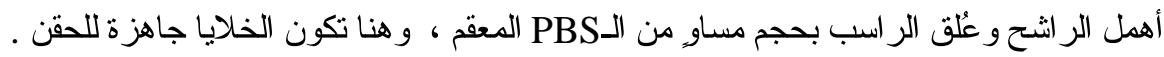

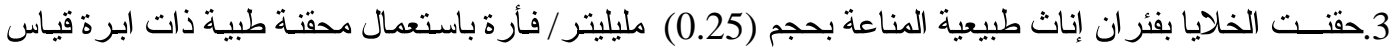

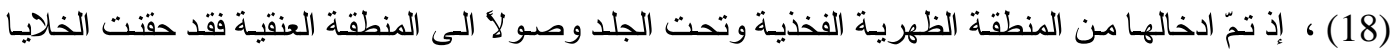
وضغطت المنطقة (ماقبل منطقة الحقن) لـنع خرو ج العالق من فتحة الحقن، ثم عقدت المنطقة .

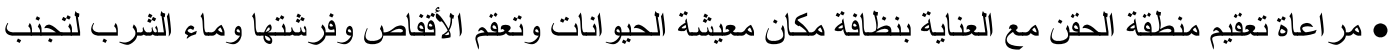
حدوث التلوث

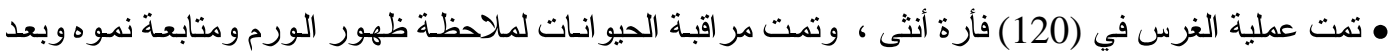

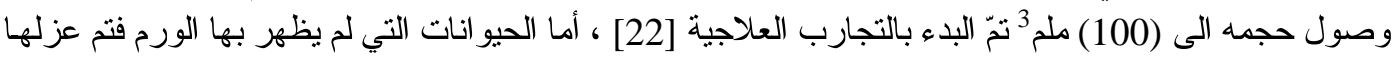


ثانياً: علاج الأورام السرطانية (المغروسة) بالكولسينات بعد عزل الفئران التي ظهر بها الورم ، تم تقسيمها الى

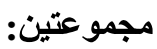

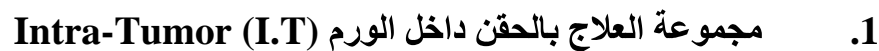

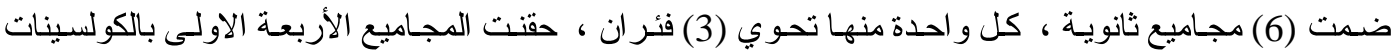

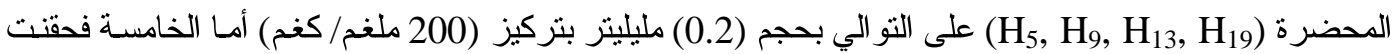

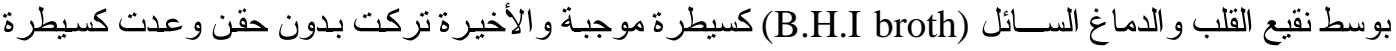

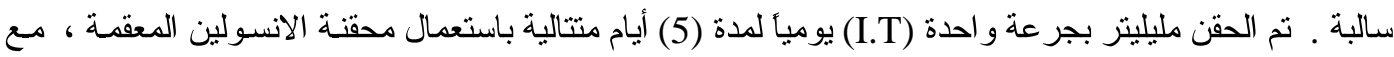

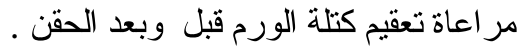

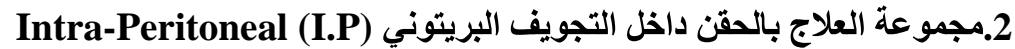

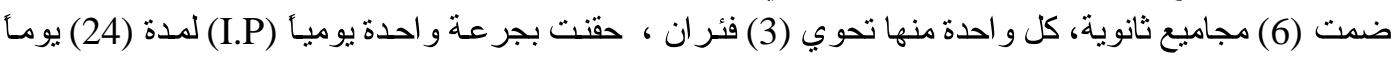

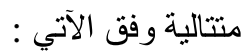
حقت المجاميع الأربعة الاولى بالكولسينات (H) H

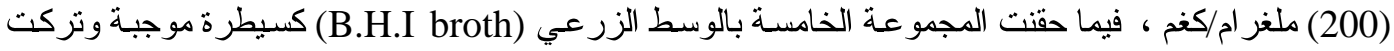
السادسة بدون حقن كسيطرة سالبة .

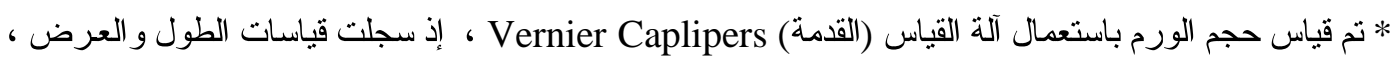
و استخر ج الحجم بالاعتماد على طريقة Ge و جماعته [23] وحسب المعادلة الآتية : $\mathrm{L}^{*} \mathrm{~W}^{2}$

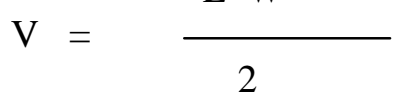

حيث ان: V V V Vجم الورم بالملم 3

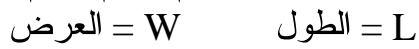

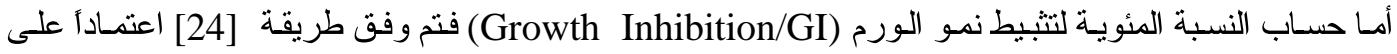
A-B

$$
\mathrm{GI} \%=
$$

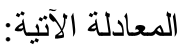

A

حيث أن: GI = النسبة المئوية لتنبيط نمو الورم. A = A

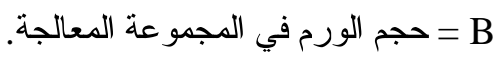
النتائج و المناقشة

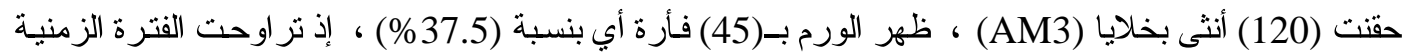

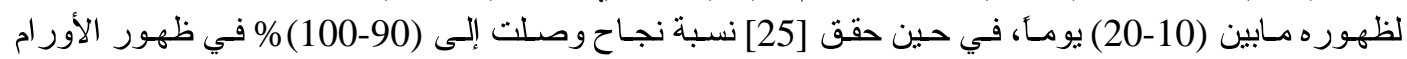

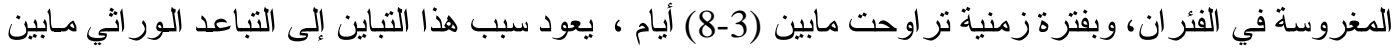

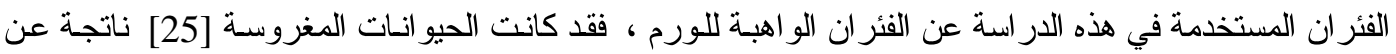

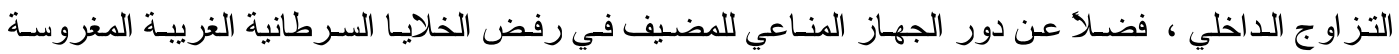

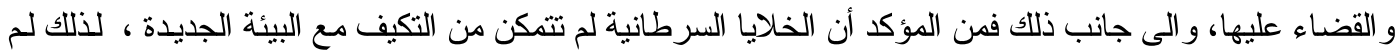

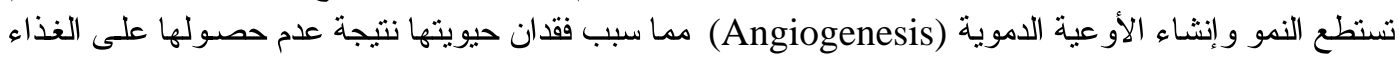

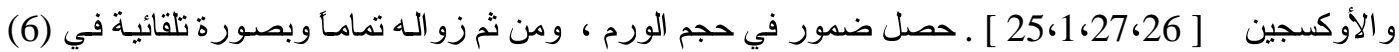

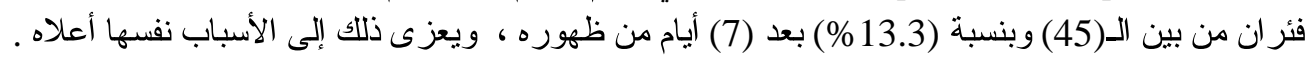

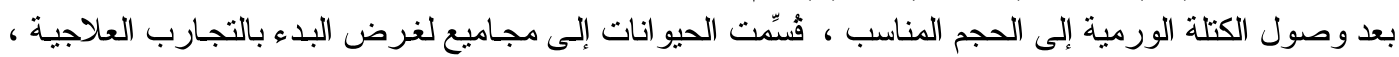

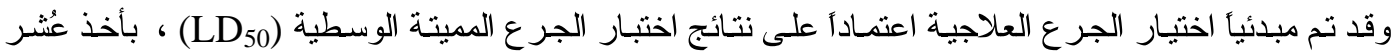

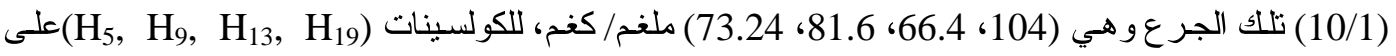

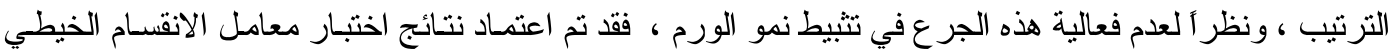

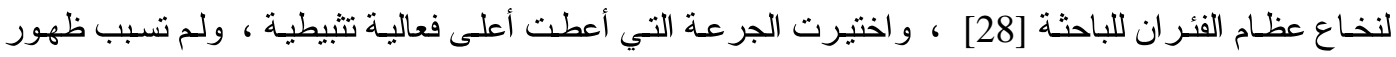

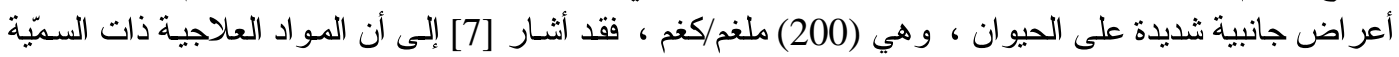


الخلوية تؤثر على الخلايا الطبيعية و السرطانية عند استخدامها بتر اكيز مرتفعة ـ ومن خلال ذلك تمّ الحصول على

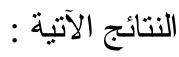

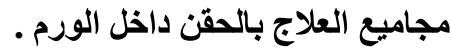

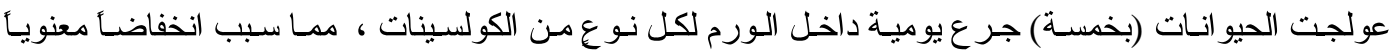

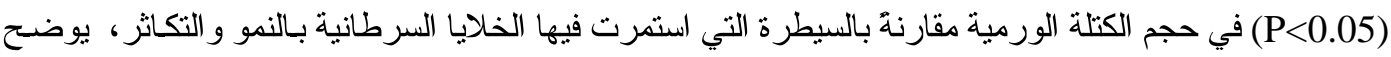

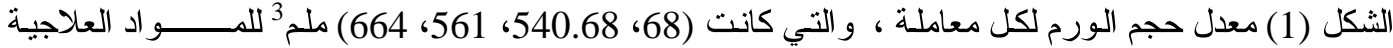

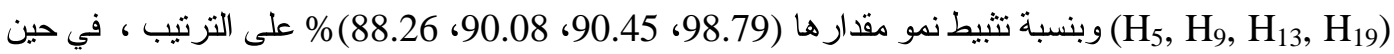

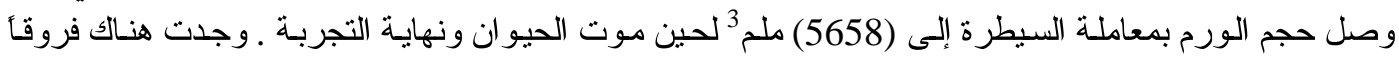

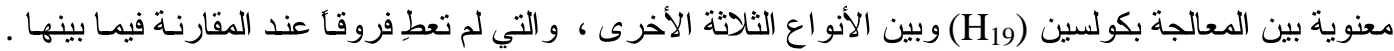

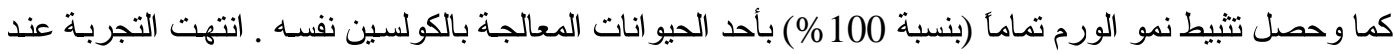

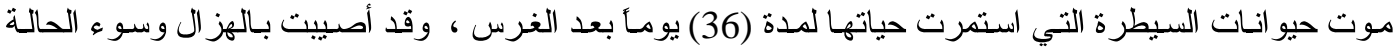

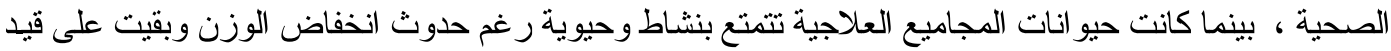

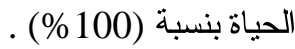

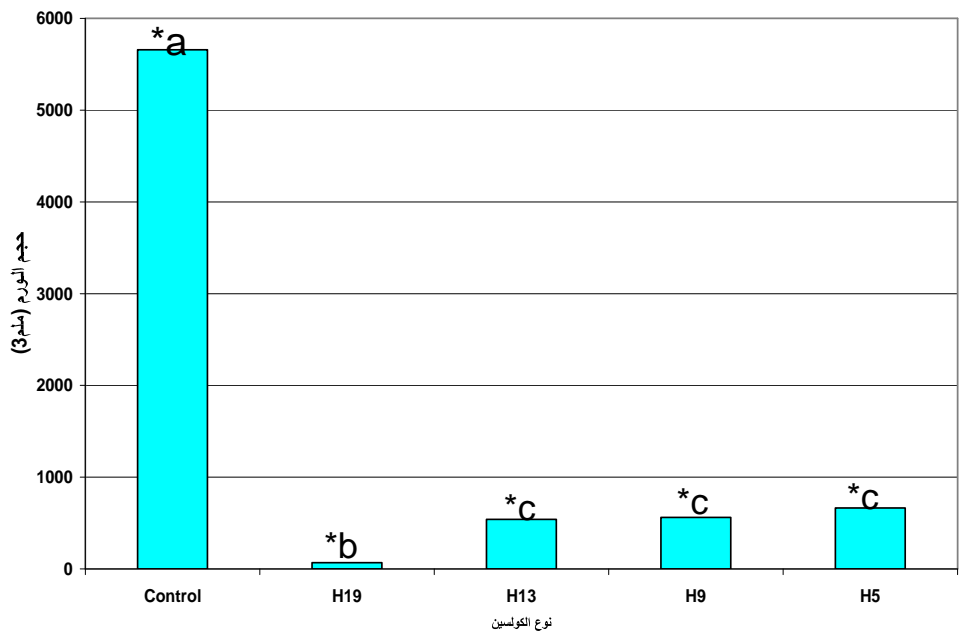

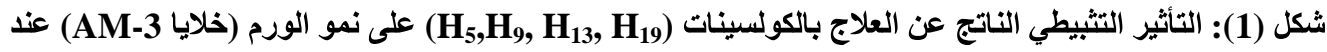

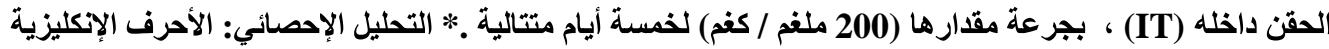
المتشابهة دلالة على عدم وجود فروق معنوية (P>0.005) بين المعاملات المختلفة

\section{مجاميع العلاج بالحقن داخل التجويف البريتوني}

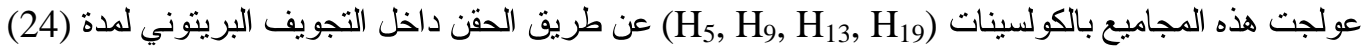

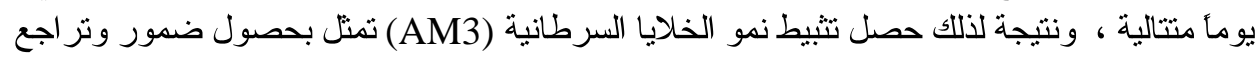

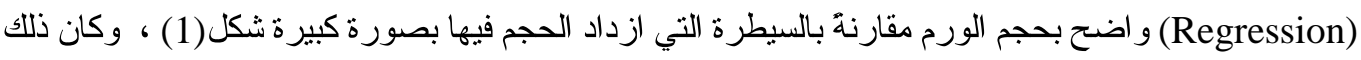

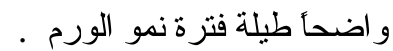



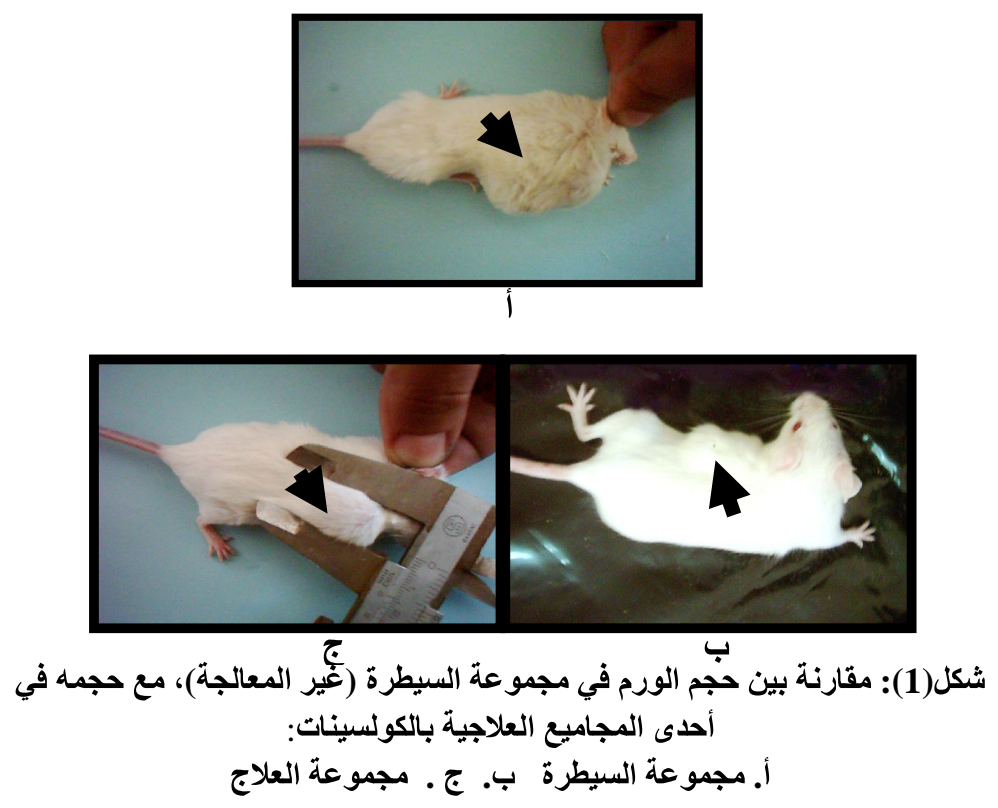

وكما هو الحال بالعلاج داخل الورم ، فقد انتهت التجربة بموت حيو انات السيطرة الذي حصل في اليوم (الخمسين)

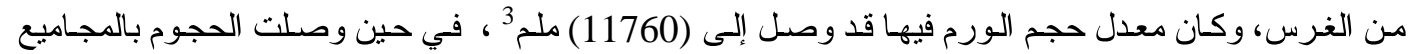

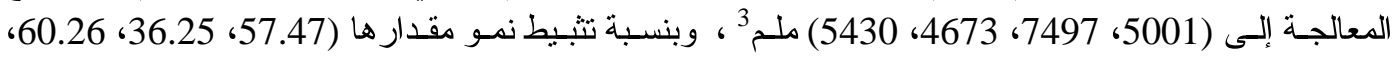
53.25\%عند المعاملة بالكولسينات (H)

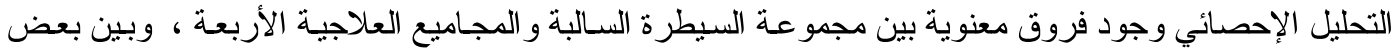

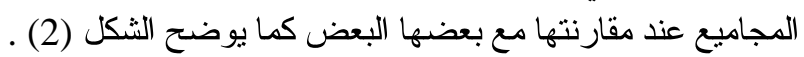

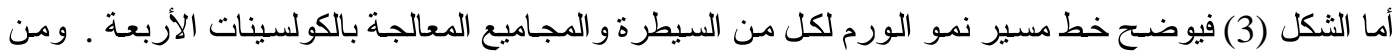

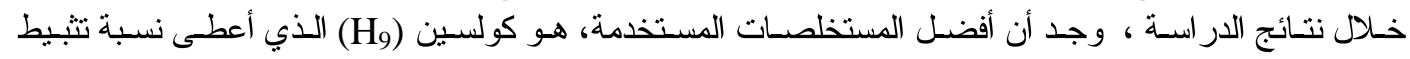

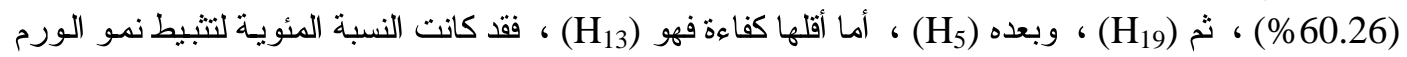

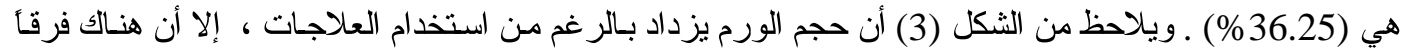

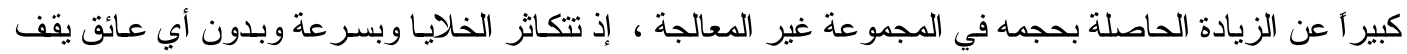

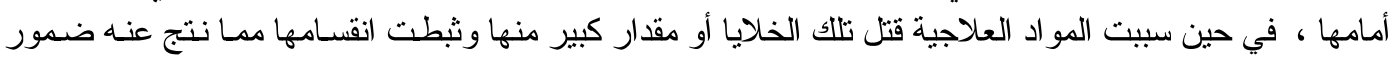

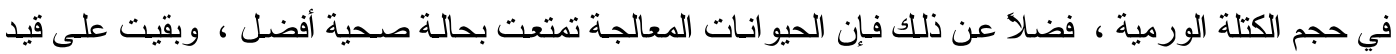

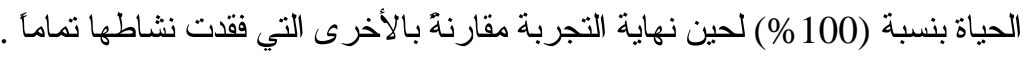

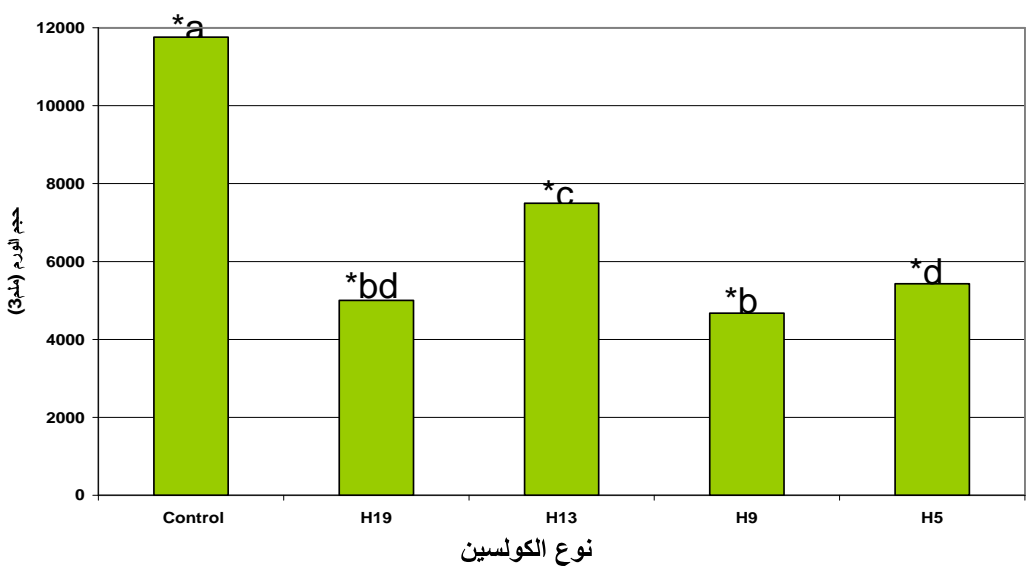

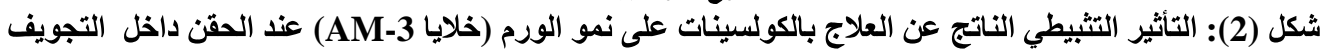
البريتوني (IP) بجرعة مقدارها (200) ملفم/ كفم من وزن الحيوان لمدة (24) يوماً متتالية . 


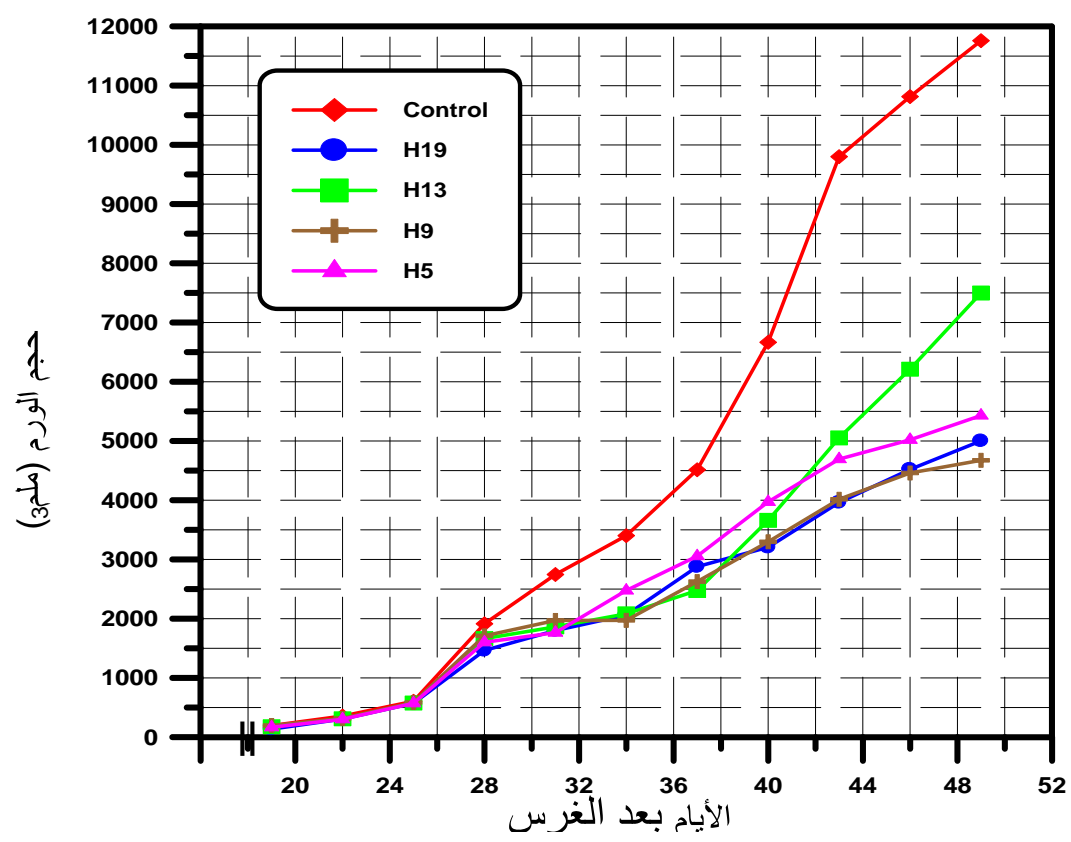

شكل (3): تأثير المعالجة بالكولسينات (H) داخل التجويف البريتوني

عند مقارنة نسب تثيط نمو الورم عند الحقن داخل الورم مع الحقن داخل البريتون ، وجد أن الدعالجة الأولى أكثر

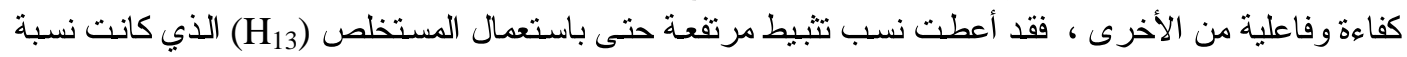
تثبيطه (36.25\%) عند الحقن (I.P) ، بينما ارتفعت إلى (I.T) (I.T) ، ربما يعزى سبب ذلك الكا

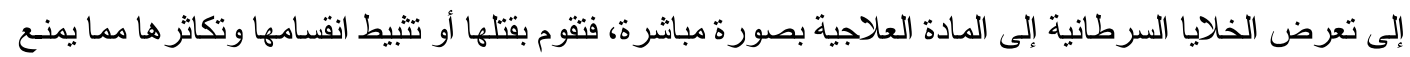

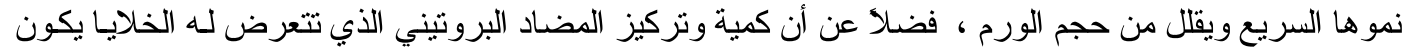

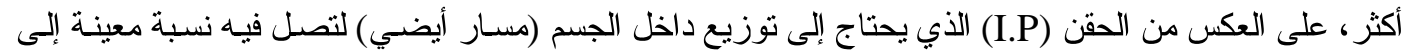

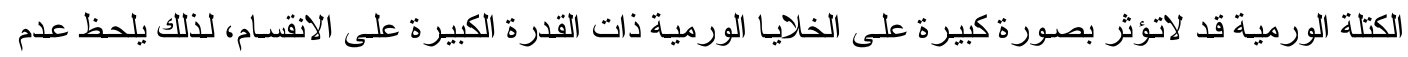

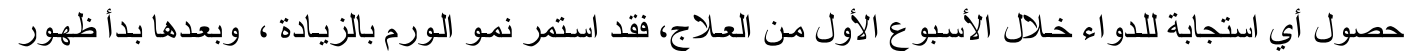

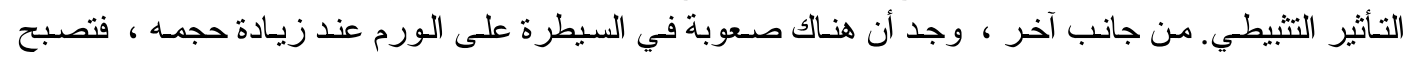

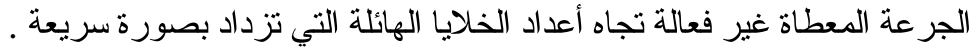

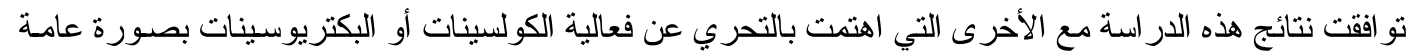

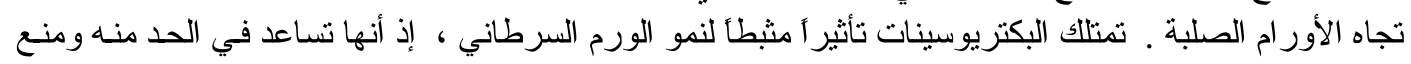

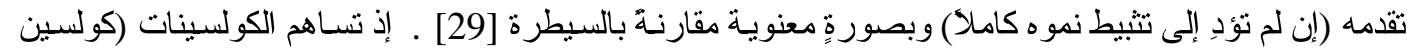

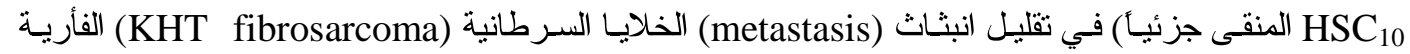

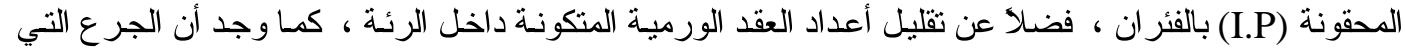

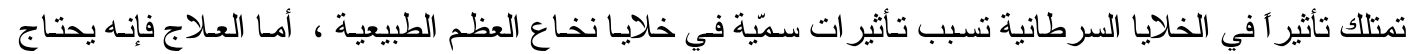

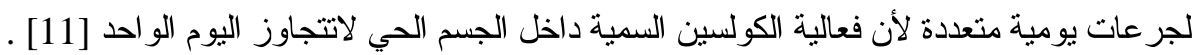

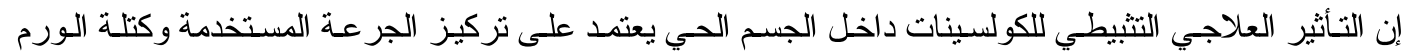

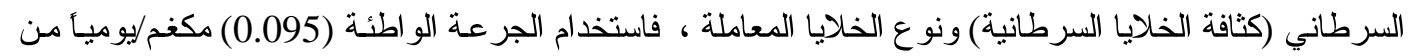

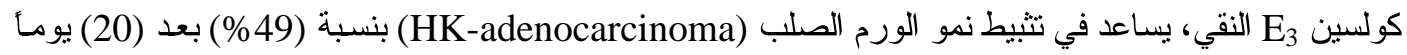

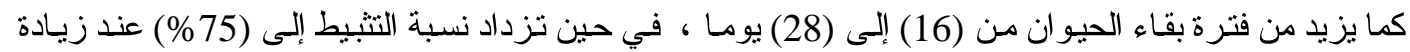

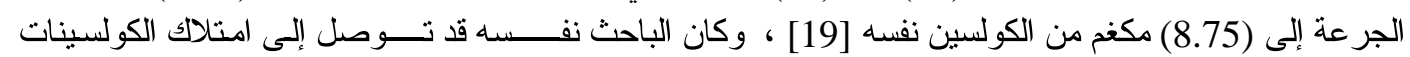

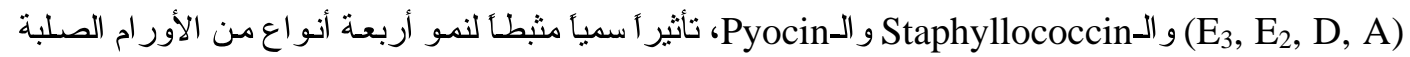


و هي وبنسبٍ مختلفة

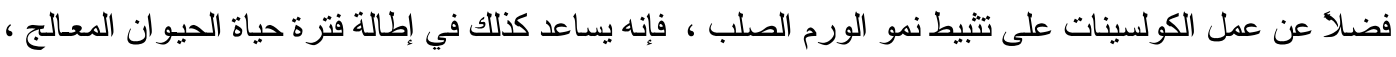

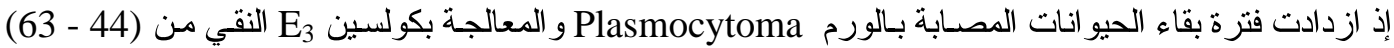

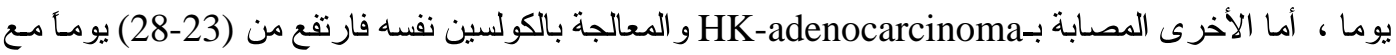

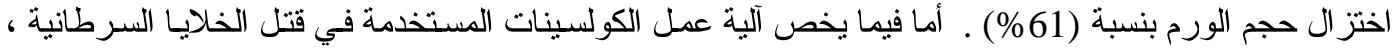

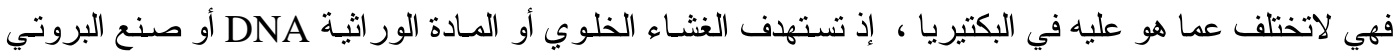
. [18،17]

من النقاط المهمة التي يجب مر اعاتها ، هو أن يتم علاج الورم وهو بحجم صغير وذللك لأن الخلايا السرطانية تكون

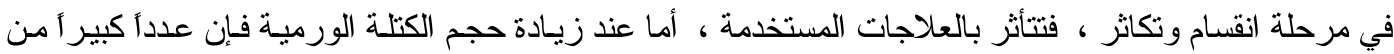

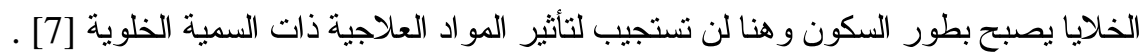

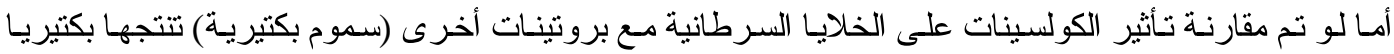

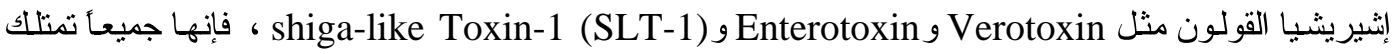

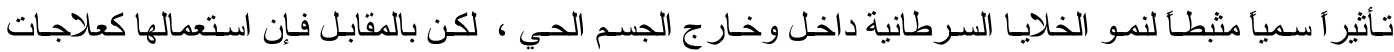

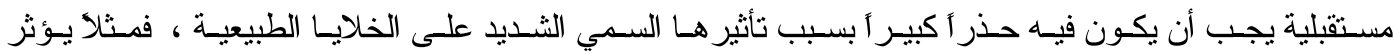

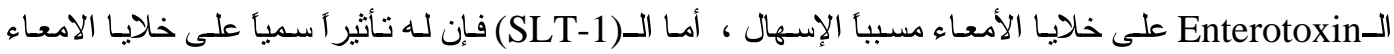

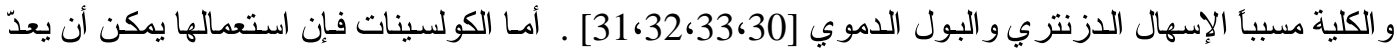

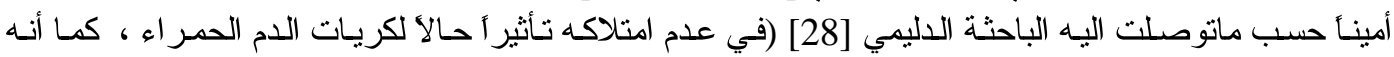

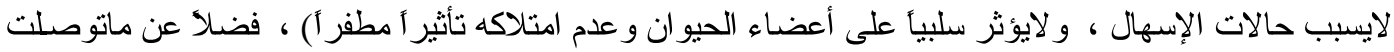

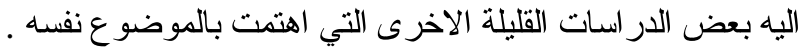

1. Nevidjon, B.M..and Sowers, K.W. 2000.A Nurse's Guide to Cancer Care, Lippincott.

2. Kumar, V.;Cotran, R.S.and Robbins,S.L.2003.Robbins Basic Pathology. $7^{\text {th }}$ ed., Saunders an Imprint of Elsevier Science.

3. Leopold, L.; Berger, M.; Cheng, S.; Giles, E. and , Estey, E.2003. Comparative efficacy and safety of Gemtuzamab Ozogamicin monotherapy and high-dose cytarabine combination therapy in patients with acute myeloid leukemia in first relapse.Clin. Adv. Hematol. Oncol., 14: 220-5.

4. Bourgaize, D.; Jewell, T. and Buiser,R. 2000. Biotechnology, Demytifiny the Concepts. $4^{\text {th }}$ ed. McGraw-Hill. New York.,pp.313-35.

5. Linenberger, M. 2005. CD33-directed therapy with gemt-Uzumab Ozogamicin in acute myeloid leukemia: progress in understanding cytotoxicity and potential mechanisms of drug resistance. Leukemia, 19: 176-82.

6. Stone, R.M. 2002. eloid Leukemia inThe Difficult Problem of Acute My the Older Adult. Cancer J. Clin.52: 363-71.

7. Trounce, J. and Gould, D. 2000.Clinical Pharmacology for Nurses. $7^{\text {th }}$ ed. CharchillLivingstone. Edinburgh. pp.275-86.

8. Giles, F.J.; Keating, A.; Goldstone, A.H.; Avivi, I.; Willman, C.L. and Kantarjian, H.M. 2002. Acute Myeloid Leukemia. Hematology ,pp. 73-110. American Society of Hem.: Education Program Book.

9. Haskell, C.M.1980. Cancer treatment. W.B. Saunders Co., Philadelphia., pp.67-75.

10. Harrey, R.A.; Champe, P.C. and Mycek, M.J.2000. Pharmacology. $2^{\text {nd }}$ ed, Lippincott Williams and Wilkins, New York. 
11. Hill, R. P. and Farkas-Himsley, H. 1991. Further Studies of The Action of a Partially Purified Bacteriocin Against a Murine Fibrosarcoma. Cancer Research, 51: 1359- 65.

12. Chumchalova, J. and Smarda, J. 2003.Human Tumor Cells are Selectively Inhibited by Colicins. Folia Microbiol., 48: 111-5.

13. Smajs, D.; Pilsl, H. and Braun, V. 1997. Colicin U, Anovel Colicin Produced by Shigella boydii. J. of Bacteriol., 179: 4919- 28.

14. Wooley, R. E.; Brown, J.; Gibbs, P. S.; Nolan, L. K. and Turner, K. R.1994. Effect of Normal Intestinal Flora of Chickens on Colonization by Visulent Colicin VProducing, Avirulent, and Mutant Colicin V- Producing Avian Escherichia coli. Avian Diseas., 38: 141-5.

15. Smajs, D. and Weinstock, G. 2001. The Iron- and lTemperature- Regulated CjrBC Genes of Shigella and Enteroinvasive Escherichia coi Strains Code for Colicin JS Uptake. J. of Bacteriology, 183: 3958-66.

16. Lazdunski, C.; Bouveret, E.; Rigal, A.; Journet, L.; Lloubes, R. and Benedett, H. 1998. Mini- Review, Colicin Import into Escherichia coli cells. J. of Bacteriol.,180: 4993- 5002.

17. Smarda, J. and Smajs, D. 1998. Colicins-Exocellular Lethal Proteins of Escherichia coli. Folia Microbiol, 43: 563-82.

18. Cursino, L.; Smarda, J.; Chartone, E. and Nascimento, A. 2002.Recent Updated aspects of Colicins of Enterobacteriaceae. Braz. J. Microbiol., 33: 196-217.

19. Smarda, J. 1992. Colicins as Anti-Tumour Drugs. Nato Asi Series, 65: 505-10.

20. Herschman, H. R. and Helinski, D. R. 1967. Purification and Characterization of Colicin $\mathrm{E}_{2}$ and Colicin $\mathrm{E}_{3}$. The J. of Biological Chemistry, 242: 5360- 8.

21. Lowry, O. H.; Rosebrough, N. J.; Farr, A. L. and Randall, R. J. 1951. Protien Measurement with the Folin Phenol Reagent. J. Biol. Chemical., 193: 265- 75.

22. Shibata, T.; Giaccia, A.J. and Brown, M. 2002. Hypoxia-Inducible Regulation of a Prodrug-Activating Enzyme for Tumor-Specific Gene Therapy. Neoplasia, 4: 40-8.

23. Ge, N.L.; Ye, S.; Zheng, N.; Sun, R.; Liu, Y. and Tang, Z. 2003. Prevention of hepatocellular carcinoma in mice by IL-2 and B7-1 genes co-transfected liver cancer cell vaccines. World J. Gastroenterol, 9: 2182-5.

24. Phuangsab, A.; Lorence, R.M.; Riechard, K.W.; Peeples, M.E. and Walter, R.J. 2001. Newcastle disease virus therapy of human tumor xenografts: anti-tumor effects of local or systemic administration. Cancer Letters, 172: 27-36.

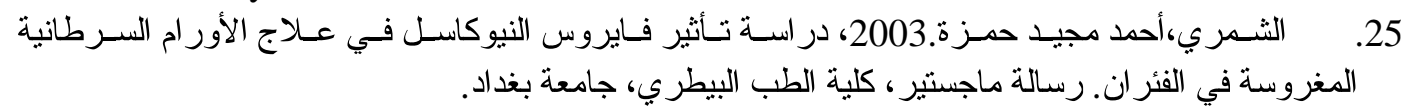

26. Ruddon, R.W. 1981. Cancer Biology. Oxford University Press, New York.

27. Roitt, I.; Brostoff, J. and Male, D. 2001. Immunology. $6^{\text {th }}$ ed., Mosby, London, New York, Toronto.

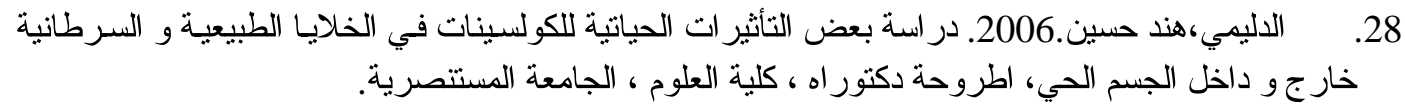

29. Fumarola, D.; Bello, P.; Palma; R. Miragliott, G. and Panaro, A. 1997. Bacteriocins as cytotoxic agents in experimental neoplasms: considerations on the role of possible contaminants. G Batteriol Virol Immunol. 70: 87-93. 
30. Farkas- Himsley, H.; Hill, R.; Rosen, B.; Arab, S. and Lingwood, C. D. 1995. The Bacterial Colicin Active Against Tumor Cells in Vitro and in Vivo is Verotoxin 1. Proc- Natl- Acad- Sci- USA. 92: 6996- 7000.

31. Arab, S.; Rutka, J. and Lingwood, C. 1999.. Verotoxin Induces Apoptosis and the Complete, Rapid, Long-Term Elimination of Human Astrocytoma Xenografts in Nude Mice. Oncology Research, 11: 33-9.

32. Gariepy, J. 2001. The use of shiga-like toxin 1 in cancer therapy. Critical Reviews in Oncology-Hematology, 39: 99-106.

33. Pitari, G.M.; Zingman, L.V.; Hodgson, (D.M.); Alekseev, A.E.; Terzic, A. and Waldman, S.A. 2003. Bacterial enterotoxins are associated with resistance to colon cancer. Proc-Natl-Acad-Sci-USA,100: 2695-9. 